\title{
Topical steroid addiction in atopic dermatitis
}

This article was published in the following Dove Press journal:

Drug, Healthcare and Patient Safety

14 October 2014

Number of times this article has been viewed

\section{Mototsugu Fukaya' \\ Kenji Sato ${ }^{2}$ \\ Mitsuko Sato ${ }^{3}$ \\ Hajime Kimata ${ }^{4}$ \\ Shigeki Fujisawa ${ }^{5}$ \\ Haruhiko Dozono6 \\ Jun Yoshizawa ${ }^{7}$ \\ Satoko Minaguchi ${ }^{8}$}

'Tsurumai Kouen Clinic, Nagoya,

${ }^{2}$ Department of Dermatology, Hannan Chuo Hospital, Osaka, ${ }^{3}$ Sato Pediatric Clinic, Osaka, ${ }^{4}$ Kimata Hajime Clinic, Osaka, ${ }^{5} \mathrm{Fujisawa}$ Dermatology Clinic, Tokyo, 'Dozono Medical House, Kagoshima, ${ }^{7}$ Yoshizawa Dermatology Clinic, Yokohama, ${ }^{8}$ Department of Dermatology, Kounosu Kyousei

Hospital, Saitama, Japan

Video abstract

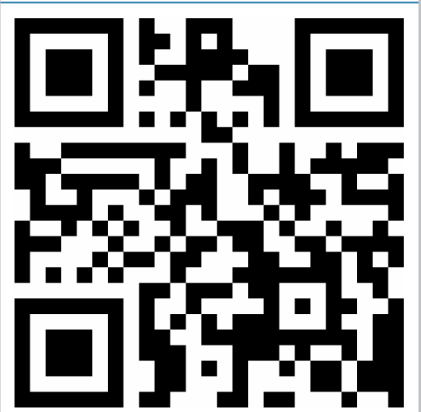

Point your SmartPhone at the code above. If you have a QR code reader the video abstract will appear. Or use: http://dvpr.es/XNuadg

Correspondence: Mototsugu Fukaya Tsurumai Kouen Clinic, 5-20-6, Chiyoda, Naka-ku, Nagoya-City, Aichi Prefecture, 460-00I2 Japan

Email moto@earth.ocn.ne.jp
Abstract: The American Academy of Dermatology published a new guideline regarding topical therapy in atopic dermatitis in May 2014. Although topical steroid addiction or red burning skin syndrome had been mentioned as possible side effects of topical steroids in a 2006 review article in the Journal of the American Academy of Dermatology, no statement was made regarding this illness in the new guidelines. This suggests that there are still controversies regarding this illness. Here, we describe the clinical features of topical steroid addiction or red burning skin syndrome, based on the treatment of many cases of the illness. Because there have been few articles in the medical literature regarding this illness, the description in this article will be of some benefit to better understand the illness and to spur discussion regarding topical steroid addiction or red burning skin syndrome.

Keywords: topical steroid addiction, atopic dermatitis, red burning skin syndrome, rebound, corticosteroid, eczema

\section{Introduction}

Although the problem of steroid phobia in patients with atopic dermatitis has sometimes been brought up for discussion in the dermatological field, most dermatologists seem to regard it as a result of simple fear, due to patient ignorance, and rarely correlate it with topical steroid addiction (TSA) or red burning skin syndrome (RBSS).

However, among doctors who have had the experience with treating this illness, there is the belief that at least some of the steroid-phobic attitude of these patients may be due to the fact that they may have already once developed TSA or RBSS.

The American Academy of Dermatology (AAD) published a new guideline regarding topical therapy in atopic dermatitis in May 2014. Although TSA or RBSS had been mentioned as possible side effects of topical steroids in a 2006 review article in the Journal of the American Academy of Dermatology, no statement was made regarding this illness in the new guidelines. ${ }^{1,2}$

According to increased requests from patients, the National Eczema Association (NEA) assembled a task force to elucidate the realities of the illness. ${ }^{3}$ As the NEA expressed the end points of its research as some interrogative sentences on its website, the authors also will try to describe the illness by answering those questions.

When placing importance on TSA or RBSS, there are three problems, from the viewpoint of preventing TSA or RBSS, in the new AAD guidelines. ${ }^{2}$ The latter part of this article discusses these problems. 


\section{The NEA questions and our answers to them How do you define steroid addiction?}

First of all, TSA should not simply be referred to as steroid addiction. More precisely, it should be expressed as a topical corticosteroid addiction. However, as the term "topical steroid" usually only refers to topical corticosteroids (TCS) in the dermatological field, the use of the term steroid for corticosteroid is acceptable. Conversely, the term steroid can mean both topical and systemic steroid (ie, oral or injection). Addiction occurs more frequently and uniquely with the topical form. Therefore, the modifier "topical" is important.

The term "addiction" to TCS was first used by Burry in Australia in $1973 .{ }^{4} \mathrm{He}$ conveyed a situation in which the patients become unable to do without the TCS because the eczema would recur soon after discontinuation. Thus, Burry's use of the term addiction was directed more toward expressing patients' behavior. Kligman and Frosch used the term addiction with a more dermatological or morphological meaning. ${ }^{5}$ They described the situation in which the symptoms became worse than pretreatment after withdrawal as addiction, and they mentioned that atopic dermatitis was a typical disease that was liable to develop addiction. In the review article by Kligman and Frosch, it was unclear whether the addiction occurred only in particular areas of the body or whether it could affect the entire body surface. Rapaport and Lebwohl, ${ }^{6}$ and Enomoto et $\mathrm{al}^{7}$ reported that the rebound phenomenon can affect the entire body surface, and Rapaport and Lebwohl ${ }^{6}$ named the illness as RBSS.

For these reasons and the characteristics of skin manifestations during the rebound period, TSA shall be defined as follows: TSA is the situation where skin develops more severe or diverse skin manifestations after the withdrawal from TCS than at preapplication.

\section{What are the clinical findings of steroid addiction?}

The clinical findings of TSA should be described separately before and after withdrawal. Before withdrawal, the addicted skin usually looks almost normal or well-controlled by TCS. Patients often subjectively notice that some effects occur; for example, the itching may be more uncomfortable than before (at the beginning of application of TCS) or the TCS do not work as well as before. In some patients, after taking TCS, prurigo-like eruptions develop or remain as intractable lesions, such as intractable nodules with severe itching. Dermatologists often explain prurigo as a chronic and difficult-to-treat type of eruption seen in patients with atopic dermatitis. However, it is often a sign of addiction (Figure 1A).

After TCS withdrawal, the erythema often develops from the area of the skin where the intractable eczema remained and spreads gradually day by day. The original area of eczema that had become thickened (often mixed with prurigo as mentioned) becomes flattened, and the border with extending erythema is obscured (Figure 1B). This rebound eruption extends to areas of the skin where TCS have never been applied. The typical spreading course of the rebound eruption extends from the face to the neck, upper extremities, trunk, and then to the lower extremities, although there may be many variants. Sometimes, the rebound eruption spreads from only one eczematous finger to the arms, trunk, face, and lower extremities and then on to the whole body, even when there had been no other eczema on the patient and TCS had been used only on the affected finger.

In milder cases, the rebound eruption simply consists of flushing or erythema with or without exudative edema, while in the more severe cases, a myriad of skin manifestations, including papules, pustules, or erosions (Figure 2), can be seen. The latter findings are sometimes accompanied by a high fever, of approximately $102^{\circ} \mathrm{F}$.

The peak of the rebound reaction also ranges, from several days to a few months depending on the patient. The rebound eruption gradually becomes dull as time passes and as it spreads. The high fever will generally subside after several days; however, the differential diagnosis including sepsis, as a complication, should never be neglected.

After the acute phase of the red exudative rebound, a dry, itchy phase follows, with thickened and desquamative skin. Patients usually become depressed and pessimistic during this period because of the symptoms and because of the fact that doctors do not know how to treat them without recommending the resumption of TCS. However, the skin gradually improves despite patient concerns but becomes very sensitive and reacts to every small stimulus (Figure 3). Even seasonal climate changes may become a burden for the sensitive skin during this period and often temporarily causes aggravation.

Excessive sweating or the occurrence of itchy wheals sometimes develops, but these are signs of recovery. The addicted skin becomes normal as time passes, and the increased sensitivity after withdrawal decreases. The entire course can take from weeks to even years.

After complete withdrawal, the skin regains its original appearance or returns to the original skin condition of atopic dermatitis. Some patients may have completely healthy skin, if the eruption that occurred before withdrawal was in fact caused by TSA rather than atopic dermatitis. 


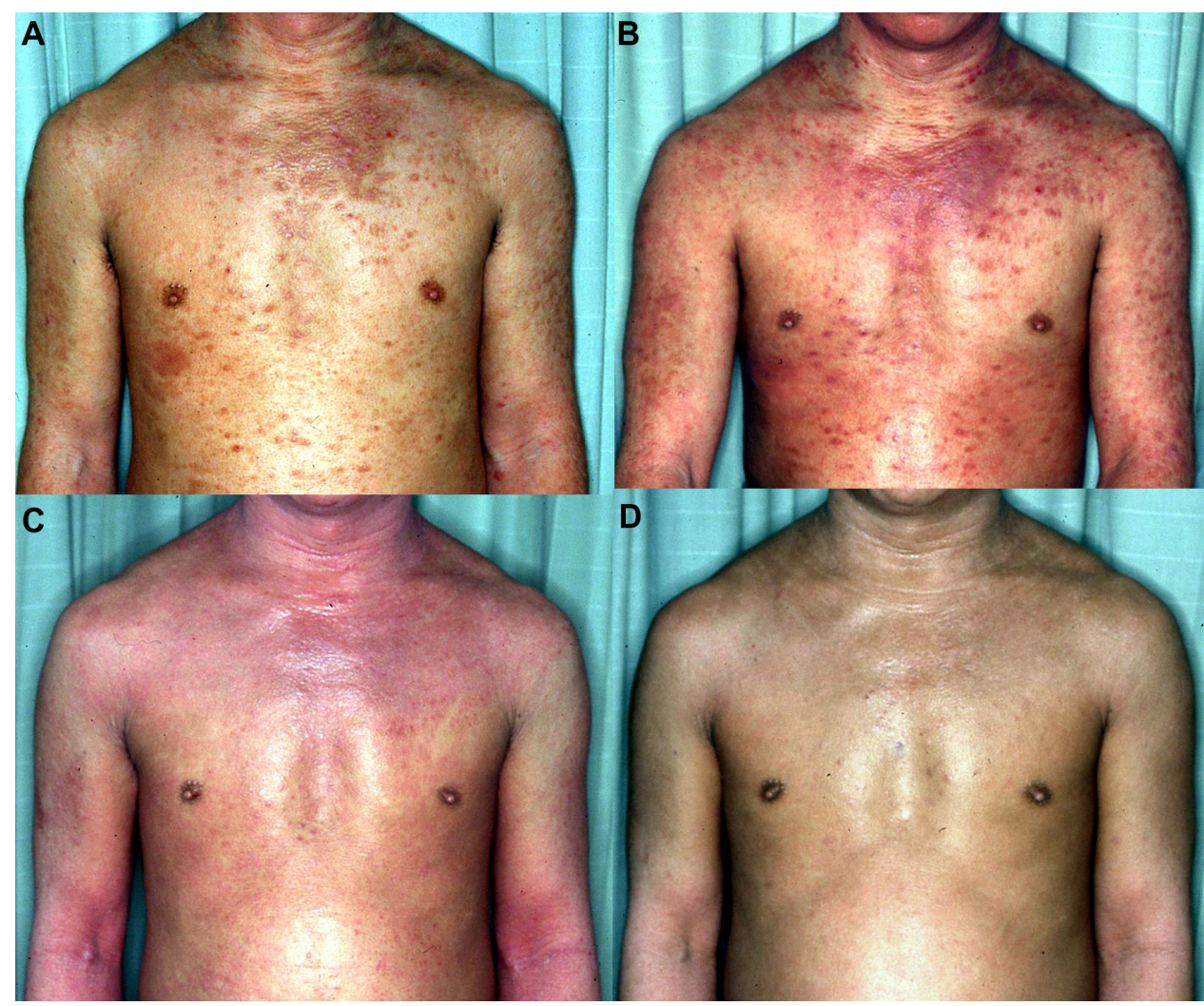

Figure I (A) A typical appearance of TSA, with prurigo-like eruption before withdrawal. (B) Appearance just after trial of decreasing the amount of potent topical steroids. The severity of addiction is so intense that the patient cannot safely withdraw by a gradual-decrease method. (C) The rebound erythema is spreading after complete cessation of steroids. (D) The appearance after I year. The rebound has almost subsided but hypersensitivity still remains.

Abbreviation: TSA, topical steroid addiction.

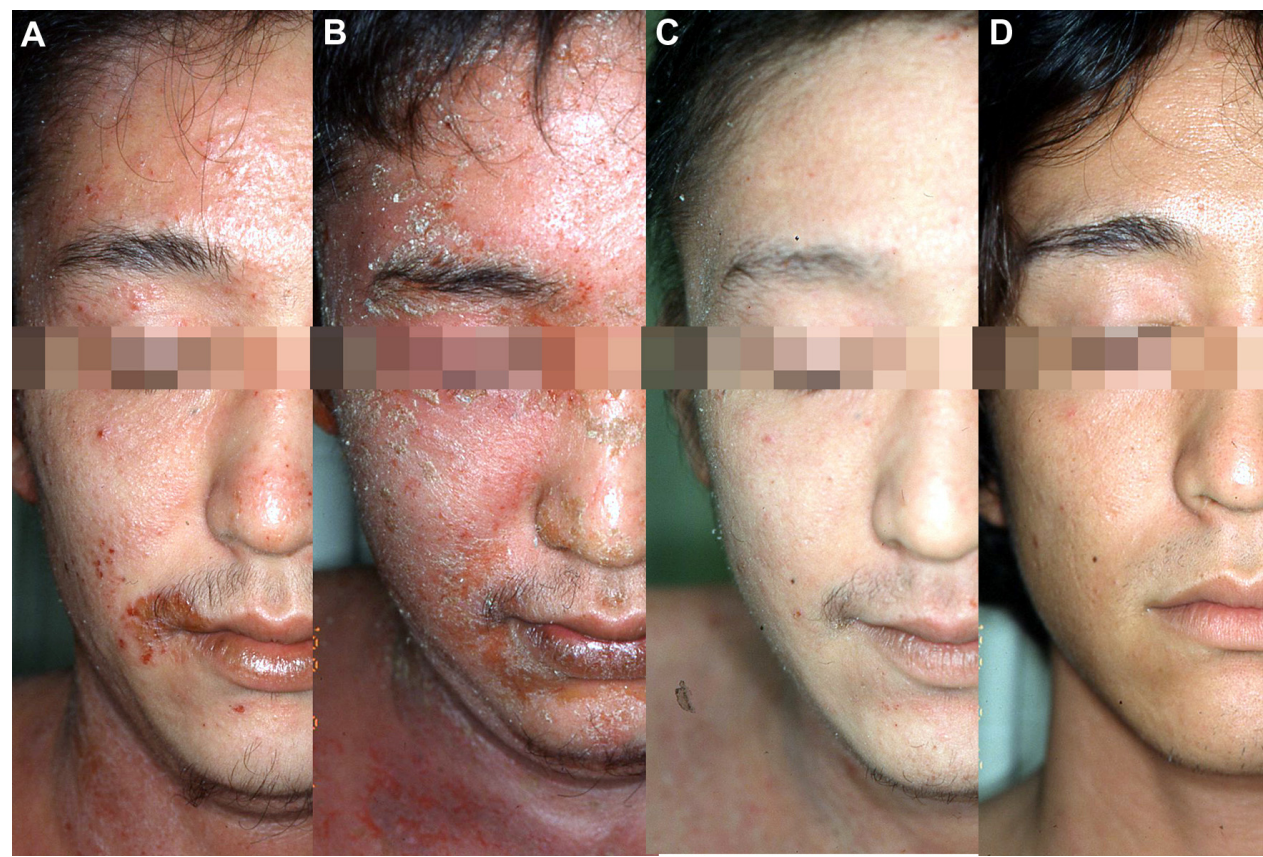

Figure 2 The procedures of withdrawal in a severe case of TSA.

Notes: (A) Before withdrawal; (B) 2 weeks after withdrawal; (C) 3 months after withdrawal; and (D) 13 months after withdrawal. Abbreviation: TSA, topical steroid addiction. 


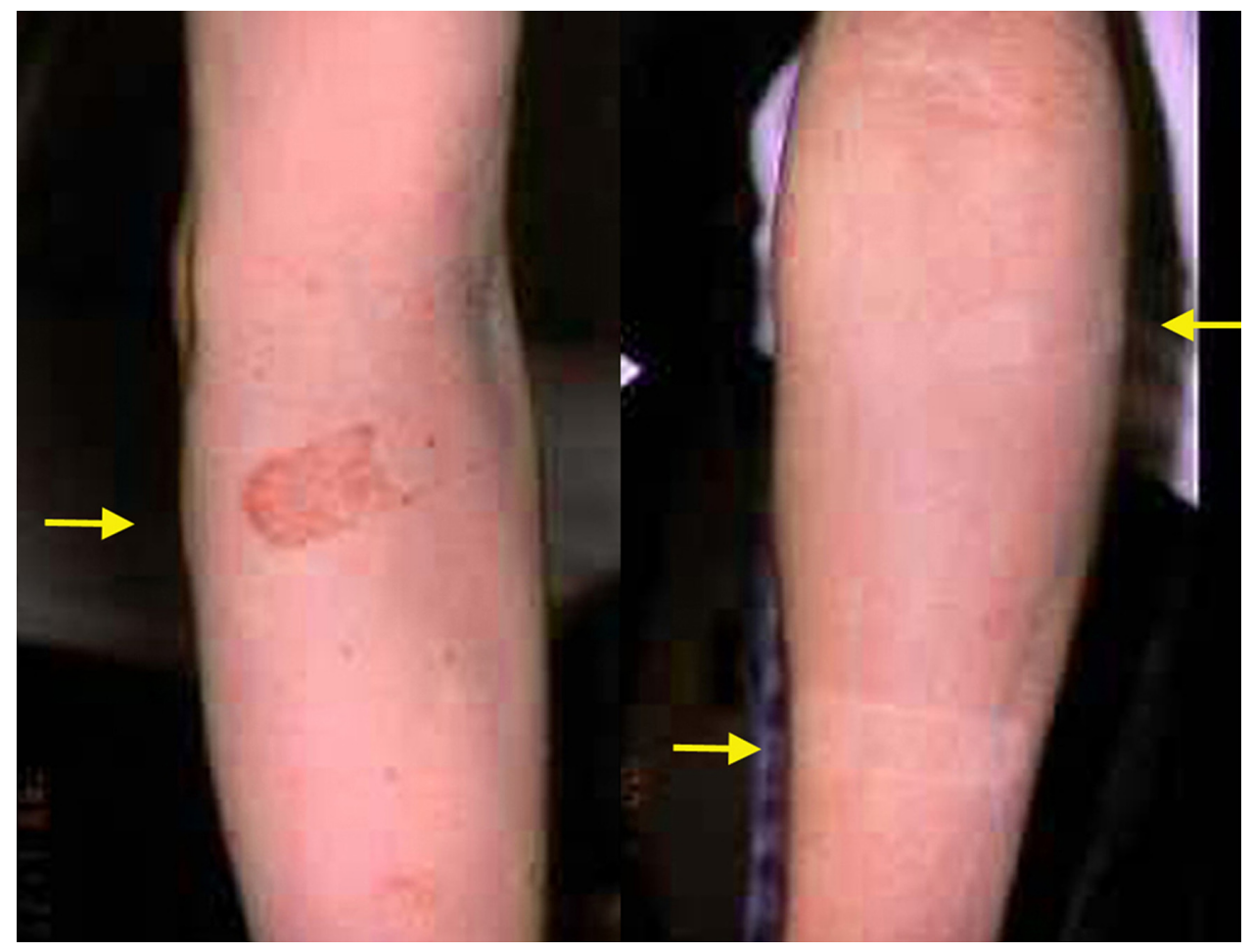

Figure 3 A case presenting hypersensitivity after topical steroid withdrawal.

Note: The skin where a band-aid was placed and detached (yellow arrows) shows an irritated appearance in the left photo, while almost normal after I year (right).

\section{What do the skin lesions look like, and how are they different to eczema?}

The skin lesion of TSA basically resembles the original skin disease. This may be one of the reasons that most doctors (particularly dermatologists) do not notice or hesitate to admit the existence of TSA. Although the appearance of the skin lesion of TSA is generally difficult to distinguish from the original skin disease, the distribution or expansion of the lesion might be a little different. The orthodox distribution of atopic dermatitis involves the neck, knees, or elbows (flexor parts of the body); in TSA, the appearance of a skin lesion is not limited to those sites. The only areas not affected by TSA or the rebound eruption are the palms and soles. The rebound eruption that extends from the central part of the body to the extremities often stops at the margin of the dorsal and palmar (or solar) sides (Figure 4). However, these can also be partially affected in severe cases.

Although the addicted skin before withdrawal may have an atrophic appearance (sometimes the skin is thin and pale similar to paper), after withdrawal, the skin temporarily becomes extraordinarily thickened. Our most intuitive description of addicted skin is, the lesions of TSA look similar to those of atopic dermatitis, but there is something different or extraordinary regarding them.

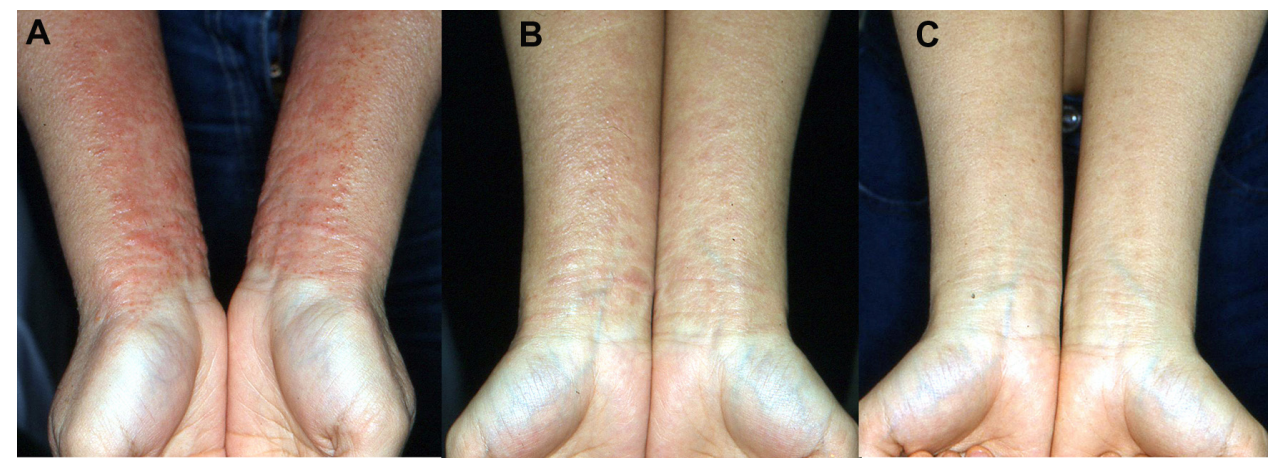

Figure 4 An example of typical demarcation around the wrists seen in the rebound eruption. Notes: (A) at the peak of rebound, (B) I month later, and (C) 2 months later. 


\section{Where on the body does it usually occur?}

Addiction can affect every part of the body. There have been many medical reports of addiction in unusual areas, such as the face, armpit, or genital area. ${ }^{8}$ The face is a special place where rosacea can occur, and the skin of the armpits or genital areas is thin. Probably this is because addiction is noticed in those areas. In other areas, the addiction progresses latently, and it may be difficult to notice until the TCS are discontinued.

Apart from the question regarding what area of the body can become addicted, the rebound eruption can extend to skin where no original lesion existed and no TCS were ever applied before. The reason is unknown, but the soles and palms are rarely affected, as already mentioned. However, as an initial addiction site, the palms or soles can also be affected. Hand eczema (or "housewives" eczema") is a typical example. Because thin skin is a factor that can cause addiction, patients with senile xerosis often develop whole-body addiction after using potent TCS.

\section{What strength of steroid and usage pattern leads to steroid addiction?}

What seems accurate is that longer periods of application and more potent strength of the TCS lead to more frequent addiction. Concrete data is very difficult to obtain because patients usually do not have a record of the applied TCS. Histologically, atrophy of the skin from TCS application becomes obvious after 6 weeks, as previously reported, ${ }^{9}$ or within 2 weeks, according to the authors' personal examination (Figure 5). Thus, it is reasonable to insist that TCS should not be used continuously for longer than 2 weeks. ${ }^{10}$ After the interruption of use for the same period as the last application of TCS, the skin may recover, and TCS can be resumed; however, there is no evidence that this kind of intermittent method of application really prevents the development of addiction.

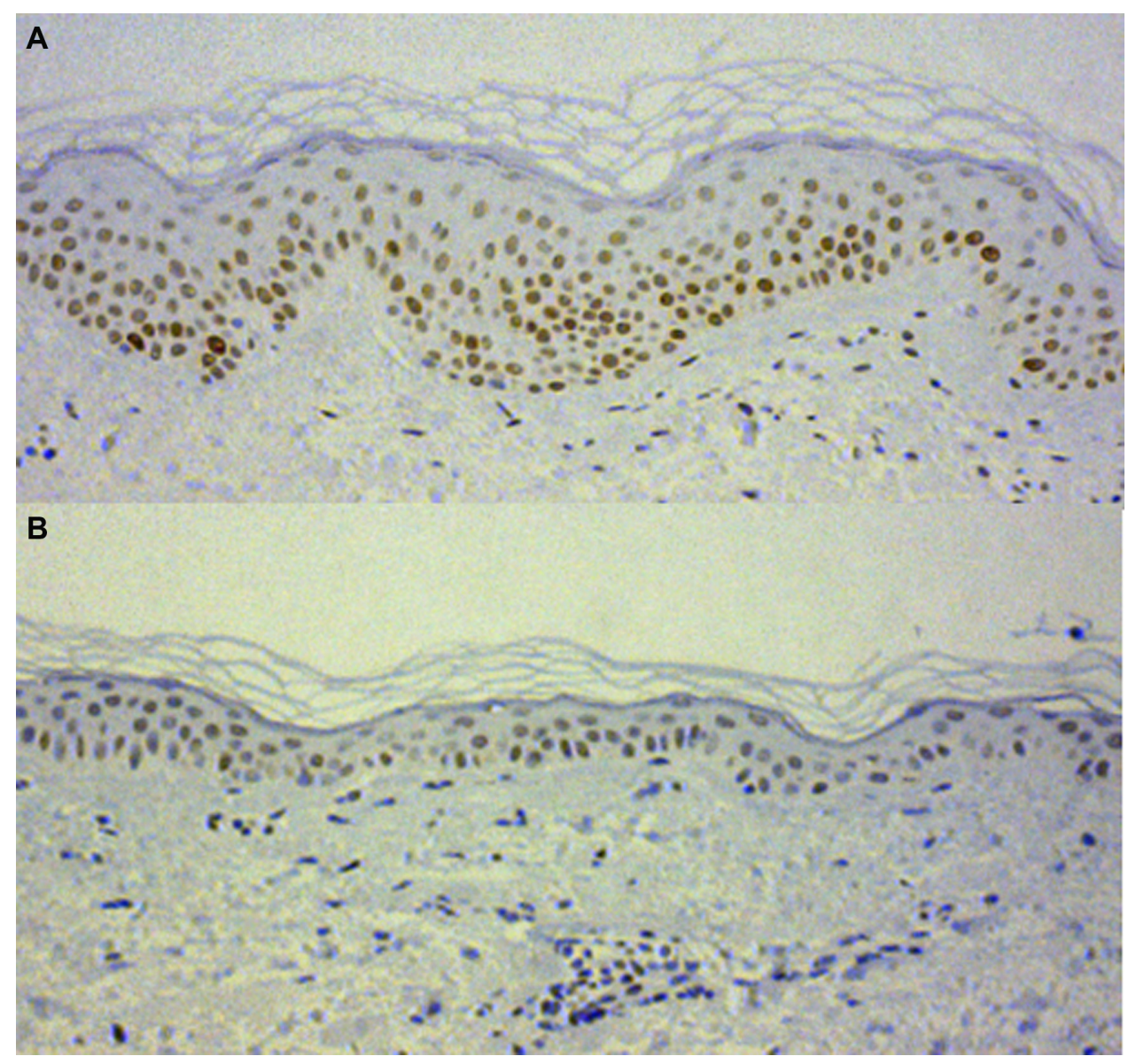

Figure $\mathbf{5}$ The result of immunohistochemical staining, using an antibody against PCNA (A) before and (B) after 2 weeks' application of $0.05 \%$ clobetasol propionate twice a day, on a healthy adult forearm.

Note: The epidermis became obviously thinner, and PCNA-positive nuclei (brown) decreased.

Abbreviation: PCNA, proliferative cell nuclear antigen. 


\section{How is steroid addiction treated?}

It goes without saying that TCS must be withdrawn in addicted patients. However, because addiction is often not recognized among dermatologists, patients cannot expect too much assistance from them. Patients often notice the abnormality of their affected skin and stop the TCS spontaneously, developing an unexpected severe rebound; only then, do the patients visit their dermatologists. Dermatologists usually only diagnose it as an aggravation of the original skin disease caused by abrupt stopping of TCS; the doctors may also strongly suggest the resumption of potent TCS and insist that TCS never suppress the hypothalamic-pituitaryadrenal axis with ordinary use. There is no relation between TSA and adrenal suppression.?

Some doctors try to switch the patient to other medications. This alternative can work if the topical steroids were completely withdrawn under the recognition that the patient was addicted. The end point of the treatment must be set as the withdrawal from TCS. However, if the situation continued to be regarded as only a simple aggravation of the initial skin disease, the TCS would usually be continued; eventually, the patient would be unable to withdraw. ${ }^{7}$

Paradoxically, the most effective agents during the rebound period are systemic steroids. The addiction seems to be caused by direct action to the skin (probably barrier destruction due to epidermis atrophy) from the TCS, while systemic steroids suppress inflammation through the internal immune system. Transient administration of systemic steroids is really effective, although most patients dislike this treatment method. There are some other empirical alternative methods to weaken the damage of the rebound eruption, but those methods work only in cases where the patient and doctor can cooperate in setting the final end point as topical steroid withdrawal. ${ }^{7}$

The difference between abrupt withdrawal and the gradual or intermittent decrease of applied steroids is small. If the patient could gradually decrease the TCS, the patient would have improved even if he or she stopped TCS cold turkey. Conversely, there are sufferers who cannot decrease the amount or potency of their TCS at all because they experience rebound immediately if the medication is decreased. Such patients are more severely addicted, and it does not make sense to advise them to gradually stop TCS. These patients are forced to stop abruptly and are generally depressed during the process. While psychological support and the prevention of complications (eg, sepsis) may be the most that doctors can offer for such patients, time may be the most beneficial "medication" for them.

\section{How common is steroid addiction syndrome?}

There are no statistics regarding the prevalence of TSA, but a survey ${ }^{11}$ conducted in Japan in 2000 may provide us with some information. In the study, ${ }^{11}$ the situations of patients with atopic dermatitis under treatment with TCS were compared, before and after 6 months of treatment. The proportion of poorly controlled disease was 19\% in adults, $10 \%$ in children, and $7 \%$ in infants. The addicted patients had to be included in the poorly controlled group, and there were fewer children than adults and very few infants. Calculated as $19 \%-7 \%=12 \%$ addicted adult patients, and $7 \%$ uncontrolled by other factors (ie, undertreatment). The numeral of $12 \%$ is smaller than $100 \%-12 \%=88 \%$, which is the proportion of estimated nonaddicted adult patients. For such patients, the warning regarding TSA does not apply and may even be harmful - if nonaddicted patients refrain from TCS for fear of TSA, they will lose an effective option for controlling their eczema. Some people may argue that spreading information regarding TSA can mislead nonaddicted patients, who comprise the majority of patients. There is some sense to this reasoning. Conversely, it is a suggestion that the information regarding TSA should always be distributed with the note that there are significantly fewer addicted patients than nonaddicted patients. On the other hand, we should not pass over the fact that the remaining $88 \%$ are also potentially addicted patients.

\section{Three problems from the viewpoint of preventing TSA or RBSS in the new AAD guidelines}

\section{The proactive approach has covert meanings}

\footnotetext{
Previously, TCS use was stopped on improvement of symptoms and signs of disease [...] However, in recent years, a more proactive approach to maintenance has been advocated for those patients who experience frequent, repeated outbreaks at the same body sites. ${ }^{2}$
}

The so-called proactive approach was described here. In the studies of proactive approach, patients' diseases were controlled by potent TCS before entry. ${ }^{12-14}$ Successfully controlled patients were divided into two groups: patients who used TCS regularly 1-2 days per week (proactive approach) and those who used TCS only when a flare developed (reactive approach). In the former, the interval between relapses was longer, and the economic burden was less. ${ }^{15}$ 
However, the study of the proactive approach may have two alternative interpretations. 1) The patients enrolled in the study were all successfully controlled by the initial treatment by potent TCS. Thus, there were patients who were judged as poorly controlled at the initial stage; those patients were not enrolled. 2) According to the proactive approach, patients cannot theoretically stop TCS forever. Eczema sufferers often heal naturally, especially infants or children. However, this natural healing tendency is disregarded in the proactive approach.

The proportion of poorly controlled group in the aforementioned studies was $10 \%-20 \%$, which is very close to the proportion of poorly controlled patients in the study of Furue et al. ${ }^{11}$ The authors accept that the proactive approach has the possibility of decreasing the number of future patients with TSA or RBSS because it is a schedule setting a cessation period of TCS. However, it must be noted that the approach does not help initially uncontrolled patients, in whom patients with TSA or RBSS may be included.

\section{Tachyphylaxis and TSA are different problems}

Development of tachyphylaxis is of concern for some practitioners, where the efficacy is thought to decrease with repeated use of the same agent, although data are lacking to suggest that this is a significant clinical problem. ${ }^{2}$

Tachyphylaxis is a medical term that expresses the phenomenon that efficacy decreases by repeated stimuli of some agent. Du Vivier and Stoughton ${ }^{16}$ reported tachyphylaxis related to TCS, in an experiment measuring vasoconstriction after consecutive application, for 4 days, of a potent TCS. The effect was improved by the cessation of TCS over the following several days. The term "tachyphylaxis" is not appropriate to represent TSA or RBSS because tachyphylaxis is usually used to faster-onset responses than TSA or RBSS.

If the term tachyphylaxis in the guidelines is used only in the original meaning - as in, the effect of epinephrine becomes weakened in the blood vessels because of frequent administration - the above quoted phrase is true. Tachyphylaxis of TCS is of little importance in clinical practice. But if the authors of the guidelines used the term tachyphylaxis to imply TSA or RBSS, the phrase "data are lacking to suggest that this is a significant clinical problem" is wrong. Although these patients may not visit dermatologists anymore and the body of medical literature is small, there are a significant number of sufferers. This is the reason why the NEA started the task force and we are now writing this article.
If the authors of the guidelines confuse tachyphylaxis with TSA or RBSS in the guidelines, it ironically articulates the fact that most dermatologists have not experienced seeing patients during withdrawal from TCS - tachyphylaxis appears prior to stopping TCS, while TSA or RBSS is the term that expresses the whole procedure of rebound or the features after withdrawal.

\section{Undertreatment should not always be regarded as inadequacy}

Although judicious use of TCS is certainly warranted, recognition of undertreatment as a result of steroid phobia is also important [...] A higher strength of recommendation (than actual level of evidence) is therefore placed on counseling, because the benefits outweigh the risks. ${ }^{2}$

The undertreatment because of steroid phobia is discussed here. There exists such steroid-phobic patients. Some patients believe their eczema will heal only if they never use TCS. In fact, this healing may happen because atopic dermatitis has a tendency of self-healing, and possibly TCS use may disturb this self-healing process. ${ }^{17}$ Moreover, when the patient becomes addicted to TCS, the recovery cannot be achieved without "undertreatment".

From short-term observation in clinical practice, up to several months or years, it certainly appears effective to treat the "undertreated" patients with TCS. For this reason, many previous studies suggest that TCS are useful. However, is it also effective from a more long-term viewpoint? Did the number of patients with adulthood atopic dermatitis increase after dermatologists began to prescribe TCS several decades ago? Why do patients with atopic dermatitis only complain or worry regarding TCS use?

Until dermatologists can clearly answer these questions, patients with atopic dermatitis have a reasonable right to choose their own therapy after receiving sufficient medical information to make an informed decision. TCS is effective, at least for short-term use, on the condition that the patients are not addicted. Therefore, the excessive warning regarding "undertreatment" may infringe on the patients' right to select treatment methods and induce the social prejudice that such patients may not be trying to treat the disease earnestly.

\section{Disclosure}

The authors report no conflicts of interest in this work.

\section{References}

1. Hengge UR, Ruzicka T, Schwartz RA, Cork MJ. Adverse effects of topical glucocorticosteroids. J Am Acad Dermatol. 2006;54(1):1-15. 
2. Eichenfield LF, Tom WL, Berger TG, et al. Guidelines of care for the management of atopic dermatitis: section 2. Management and treatment of atopic dermatitis with topical therapies. J Am Acad Dermatol. 2014;71(1):116-132.

3. nationaleczema.org [homepage on the Internet]. NEA forms Scientific Advisory Committee Task Force to study topical steroid addiction. National Eczema Association; 2014. Available from: http:// nationaleczema.org/nea-forms-scientific-advisory-committee-taskforce-study-topical-steroid-addiction/. Accessed August 20, 2014.

4. Burry JN. Topical drug addiction: adverse effects of fluorinated corticosteroid creams and ointments. Med J Aust. 1973;1(8):393-396.

5. Kligman AM, Frosch PJ. Steroid addiction. Int J Dermatol. 1979;18(1): 23-31.

6. Rapaport MJ, Lebwohl M. Corticosteroid addiction and withdrawal in the atopic: the red burning skin syndrome. Clin Dermatol. 2003;21(3): 201-214.

7. Enomoto T, Arase S, Shigemi F, Takeda K. [Steroid withdrawal syndrome by topical corticosteroid]. Journal of Japanese Cosmetic Science Society. 1991;15:117-124. Japanese.

8. Narang T, Kumaran MS, Dogra S, Saikia UN, Kumar B. Red scrotum syndrome: idiopathic neurovascular phenomenon or steroid addiction? Sex Health. 2013;10(5):452-455.

9. Zheng PS, Lavker RM, Lehmann P, Kligman AM. Morphologic investigations on the rebound phenomenon after corticosteroid-induced atrophy in human skin. J Invest Dermatol. 1984;82(4):345-352.

10. gopetition.com [homepage on the Internet]. Label all topical steroid cremes for potential severe addiction after two weeks use [petition published by Joey Brown]. GoPetition; 2011. Available from: http:// www.gopetition.com/petitions/label-all-topical-steroid-cremes-ofpotential-severe-ad.html. Accessed August 20, 2014.
11. Furue M, Terao H, Rikihisa W, et al. Clinical dose and adverse effects of topical steroids in daily management of atopic dermatitis. Br J Dermatol. 2003;148(1):128-133.

12. Van Der Meer JB, Glazenburg EJ, Mulder PG, Eggink HF, Coenraads PJ. The management of moderate to severe atopic dermatitis in adults with topical fluticasone propionate. The Netherlands Adult Atopic Dermatitis Study Group. Br J Dermatol. 1999;140(6): 1114-1121.

13. Berth-Jones J, Damstra RJ, Golsch S, et al; Multinational Study Group. Twice weekly fluticasone propionate added to emollient maintenance treatment to reduce risk of relapse in atopic dermatitis: randomised, double blind, parallel group study. BMJ. 2003;326(7403):1367.

14. Wollenberg A, Reitamo S, Girolomoni G, et al; European Tacrolimus Ointment Study Group. Proactive treatment of atopic dermatitis in adults with $0.1 \%$ tacrolimus ointment. Allergy. 2008;63(7): $742-750$.

15. Healy E, Bentley A, Fidler C, Chambers C. Cost-effectiveness of tacrolimus ointment in adults and children with moderate and severe atopic dermatitis: twice-weekly maintenance treatment vs. standard twice-daily reactive treatment of exacerbations from a third party payer (UK National Health Service) perspective. $\mathrm{Br} J$ Dermatol. 2011;164(2):387-395.

16. du Vivier A, Stoughton RB. Tachyphylaxis to the action of topically applied corticosteroids. Arch Dermatol. 1975;111(5):581-583.

17. Pampura AN. Prevalence of atopic diseases and the use of topical corticosteroids. Is there any connection? Med Hypotheses. 2005;64(3): $575-578$.
Drug, Healthcare and Patient Safety

\section{Publish your work in this journal}

Drug, Healthcare and Patient Safety is an international, peer-reviewed open-access journal exploring patient safety issues in the healthcare continuum from diagnostic and screening interventions through to treatment, drug therapy and surgery. The journal is characterized by the rapid reporting of reviews, original research, clinical, epidemiological and

\section{Dovepress}

post-marketing surveillance studies, risk management, health literacy and educational programs across all areas of healthcare delivery. The manuscript management system is completely online and includes a very quick and fair peer-review system. Visit http://www.dovepress.com/ testimonials.php to read real quotes from published authors. 